\title{
Human-Robot Dichotomy
}

\author{
Karolina Zawieska \\ Centre for Computing and Social \\ Responsibility \\ De Montfort University \\ Leicester, United Kingdom \\ karolina.zawieska@dmu.ac.uk
}

\author{
Jessica Sorenson \\ School of Education \\ Aarhus University \\ Copenhagen, Denmark \\ jeso@edu.au.dk
}

\author{
Kate Davis \\ Centre for Computing and Social \\ Responsibility \\ De Montfort University \\ Leicester, United Kingdom \\ kate.davis@dmu.ac.uk
}

\author{
Cathrine Hasse \\ School of Education \\ Aarhus University \\ Copenhagen, Denmark \\ caha@edu.au.dk
}

\author{
Alejandra Gomez \\ Ab.Acus Srl \\ Milan, Italy \\ alejandragomez@ab-acus.com
}

\begin{abstract}
This paper belongs to the area of roboethics and responsible robotics. It discusses the conceptual and practical separation of humans and robots in designing and implementing robots into realworld environments. We argue here that humans are often seen as a component that is only optional in design thinking, and in some cases even an obstacle to the success of robot performance. Such an approach may vary from viewing humans as a factor that does not belong to the robotics domain, through attempts to 'adjust' humans to robot requirements and to the overall replacement of humans with robots. Such separation or exclusion of humans poses serious ethical challenges, including the very exclusion of ethics from our thinking about robots.
\end{abstract}

\section{KEYWORDS}

Responsible Robotics, Roboethics, Ethnographic Research, Human-Robot Dichotomy

\section{ACM Reference format:}

Karolina Zawieska, Jessica Sorenson, Cathrine Hasse, Sebastian Madsen, Kate Davis, and Alejandra Gomez. 2019. Human-Robot Dichotomy. In Proceedings of HAI2019 Conference, Kyoto, Japan, 2 pages. https://doi.org/

\section{Introduction}

As robotic technologies advance, there has been a growing interest in integrating different types of robots into our society. In practice, however robotic systems and robot end-users/affected stakeholders

Permission to make digital or hard copies of part or all of this work for personal or classroom use is granted without fee provided that copies are not made or distributed for profit or commercial advantage and that copies bear this notice and the full citation on the first page. Copyrights for third-party components of this work must be honored. For all other uses, contact the owner/author(s). HAI2019, 6-10 October 2019, Kyoto, Japan.

(C) 2019 Copyright held by the owner/author(s). 978-1-4503-0000-0/18/06 $\$ \$ 15.00$ https://doi.org/ often remain separate, whether deliberately or not. Such an approach presentss serious ethical questions on the role and importance of human beings in our increasingly roboticized world. The following sections provide an overview of the findings on the human-robot dichotomy identified within the EU-funded project REELER: Responsible Ethical Learning with Robotics (see Acknowledgements). As part of the project, more than 10 researchers conducted ethnographic research on robot design, development, and implementation, along with related ethical challenges. The project works from a 'multi-variation' approach, first seeking variation in robot types, geographical places, and types of organisations, followed by seeking patterns across variations (see [1]). The main data-collection method was in-depth interviews, with both robot developers and end-users/affected stakeholders. In total, 132 interviews were conducted with participants from 8 different sectors in 13 different European countries. Excerpts from these interviews are used here to illustrate the key arguments.

\section{Human-Robot Dichotomy}

As discussed below, separation of humans and robots or explicit prioritization of robots over human beings ('either/or' approach) may take different forms and occur at different stages of robot development and implementation.

\subsection{Discipline-specific conceptualizations}

Many of the robot developers interviewed in REELER considered humans a separate domain from robots and robotics.

"Ethical challenges? You mean in terms of people? I guess that's more of a difficult one for me in the sense that the nature of what I do is to sell automation solutions to customers... That's what I'm focused on, rather than perhaps the people aspects of robotics." (Tommy, automation sales manager, material handling)

The conceptual separation of human and machine allows for a problem field to become artificially bounded to particular technical 
spheres of practice, excluding ethics and the human domain as unrelated, irrelevant, or outside of one's expertise.

\subsection{Robot-first}

These dichotomized conceptualizations can include the assimilation of the human into the robotic systems thinking. Some robot developers tend to apply to humans the same terms and concepts they apply to robots, where humans may or may not be added to the analytical frameworks for robot design. In this sense, consideration of human beings is viewed as only optional.

"My topic is robots, but for me_[laughs]. Perhaps it's not really_ In terms of ethics, perhaps it's not very good, but, you know, it [the human] is sort of like a robot --it is a resource." (Nicolas, programmer, manufacturing company)

The conflation of human and robot often comes from a prioritization of the robot over the human, both in thinking and in practice. Although there has been a recent push for collaborative robots, this project's ethnographic data shows that across different industries, some robot developers dedicate a lot of energy to preventing humans from 'spoiling' the system performance and decreasing the system efficiency.

"I put a laser scanner that detects when the operator is coming close to the robot. But I always say, why does the robot have to detect the human operator? Why can't a human operator, knowing that there is a robot that is working, stay far away? ...As soon as the operator is arriving, the robot is slowing down...This means the robot will never finish the work." (Emilia, $R \& D$ director, robotics company)

Another way robot-first thinking is applied is by making humans subservient to robots. The main factors here are the robot's role in increasing efficiency and profitability of work, and the presumption that artificial intelligence (AI) makes robots better at decisionmaking than humans. Choosing between robot and human performance rather than integrating the two raises serious concerns with regard to technology dependence, as well as the nature of human supervision of robotic and AI systems: Is it a human or a machine that is actually in control?

\subsection{Robot-only}

Extreme prioritization of robots may lead to the omission of humans from developers' conceptual frameworks but also to the lack of end-user involvement in the design and development processes, and the elimination of the human from robotized spaces. The introduction of robots to different sectors often involves an 'either/or' approach, choosing either humans or robots, and separating humans from robotic systems at the workplace.

"If you want to have result, if you want to have performance from the robot, you have to separate people from robots." (Alph, CEO, Robotics start-up)

This robot-only thinking applies even to collaborative robots that in principle are supposed to work together with humans. In media presentations we sometimes see humans and robots depicted as partners or co-workers who collaborate on a task. In practice, however, due to technological and other constraints, collaboration between robots and human workers often implies sharing time and space while executing different tasks in parallel.

In some cases humans are not considered at all, because humans are viewed as simply irrelevant or due to the difficulty in addressing the complexity of human features and behaviours (e.g., when simulating robots and robot work stations). One major concern here is the situation where a choice between humans and robots might ultimately lead to technological displacement.

"P: Generally speaking, if we put too many robots, we can have some conflict with humans.

I: Hmm. Why do you think so?

P: Why? Because for most of the people, we want to deploy robots not to be more efficient but to replace humans with robots." (Nicolas, programmer, manufacturing company)

Robot-only thinking and robot-only solutions contribute to real societal problems (e.g., unemployment, deskilling), and to fears of replacement and resistance to introduction of robots.

\section{Conclusions}

From the ethical perspective, separating humans and robots, both conceptually and practically, or prioritizing robots over humans, ultimately implies excluding ethics from robotics.

"Ethics, that is something about... That is something that I am thinking about like, more with humans, in any case. What is ethically correct and_That is mostly what I think of. It might not make me think of robots." (Eric, operator and production technologist, robot $R \& D$ company)

We argue here that in order to pursue responsible robotics we must revise the way we think and talk about human-robot systems. This includes improving our understanding of how humans and their agency can become better understood by robot developers. (Ethnographic research, e.g., shows that when humans 'spoil' system performance they have motives which are often not conveyed to or understood by robot developers.) Rather than apply dichotomized approaches, we must develop terms and perspectives that better grasp the complexity of the human-robot systems and that ultimately prioritise human well-being.

\section{ACKNOWLEDGMENTS}

This work is part of the REELER project (Responsible Ethical Learning with Robotics) that has received funding from the European Union's Horizon 2020 research and innovation programme under grant agreement No 731726 (for more information see www.reeler.eu).

\section{REFERENCES}

[1] C. Hasse (2019). The multi-variation approach. Paladyn, Journal of Behavioral Robotics, 10(1), pp. 219-227. Retrieved on 7 July 2019, doi: 10.1515/pbjr-20190017. Journal Title, 10(3), 1-10. 